\title{
VLSI FOR \\ NEURAL NETWORKS AND \\ ARTIFICIAL INTELLIGENCE
}




\section{VLSI FOR \\ NEURAL NETWORKS AND \\ ARTIFICIAL INTELLIGENCE}

Edited by

JOSÉ G. DELGADO-FRIAS

State University of New York at Binghamton

Binghamton, New York

and

WILLIAM R. MOORE

Oxford University

Oxford, United Kingdom

Springer Science+Business Media, LLC 


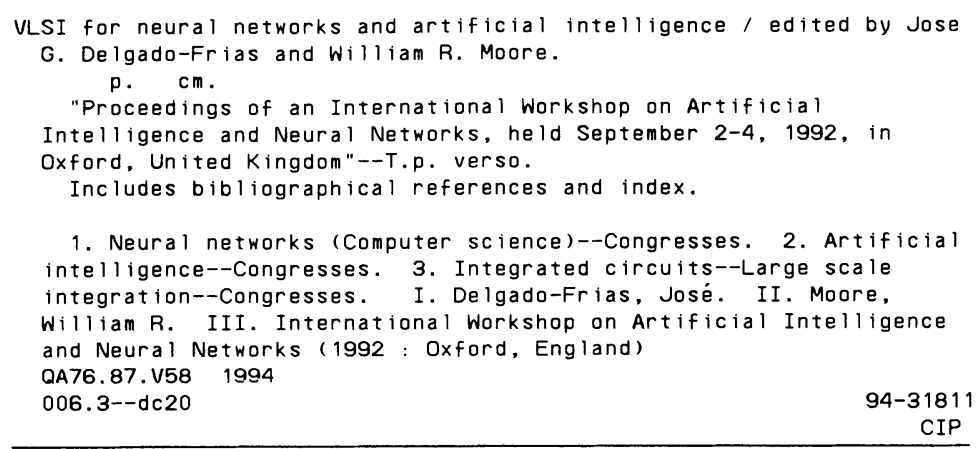

\section{ISBN 978-1-4899-1333-3 ISBN 978-1-4899-1331-9 (eBook) \\ DOI 10.1007/978-1-4899-1331-9}

Proceedings of an International Workshop on Artificial Intelligence and Neural Networks, held September 2-4, 1992, in Oxford, United Kingdom

(C) 1994 Springer Science+Business Media New York

Originally published by Plenum Press, New York in 1994.

Softcover reprint of the hardcover 1st edition 1994

\section{All rights reserved}

No part of this book may be reproduced, stored in a retrieval system, or transmitted in any form or by any means, electronic, mechanical, photocopying, microfilming, recording, or otherwise, without written permission from the Publisher 


\section{PROGRAMME COMMITTEE}

Nikolaos Bourbakis, State University of New York at Binghamton, USA Howard Card, University of Manitoba, Canada

José Delgado-Frias, State University of New York at Binghamton, USA

Simon Lavington, Univeristy of Essex, $U K$

Will Moore, University of Oxford, UK

Alan Murray, University of Edinburgh, UK

Lionel Tarassenko, University of Oxford, UK

Stamatis Vassiliadis, IBM, USA

Michel Weinfield, EP Paris, France 


\section{PREFACE}

Neural network and artificial intelligence algorithms and computing have increased not only in complexity but also in the number of applications. This in turn has posed a tremendous need for a larger computational power that conventional scalar processors may not be able to deliver efficiently. These processors are oriented towards numeric and data manipulations. Due to the neurocomputing requirements (such as non-programming and learning) and the artificial intelligence requirements (such as symbolic manipulation and knowledge representation) a different set of constraints and demands are imposed on the computer architectures/organizations for these applications.

Research and development of new computer architectures and VLSI circuits for neural networks and artificial intelligence have been increased in order to meet the new performance requirements. This book presents novel approaches and trends on VLSI implementations of machines for these applications. Papers have been drawn from a number of research communities; the subjects span analog and digital VLSI design, computer design, computer architectures, neurocomputing and artificial intelligence techniques.

This book has been organized into four subject areas that cover the two major categories of this book; the areas are: analog circuits for neural networks, digital implementations of neural networks, neural networks on multiprocessor systems and applications, and VLSI machines for artificial intelligence. The topics that are covered in each area are briefly introduced below.

\section{Analog Circuits for Neural Networks}

Using analog circuits as a mean to compute neural networks offers a number of advantages such as smaller circuit size, higher computing speed, and lower power dissipation. Neural network learning algorithms may need be implemented directly in analog hardware in order to reduce their computing time. Card presents a number of analog circuits for on-chip supervised and unsupervised learning. Landolt has developed and implemented in analog CMOS a full Kohonen network with learning capability. Valle et al report an implementation of a backpropagation learning algorithm along with some simulation results. Lafargue et aladdress the problem of designing a mixed analog-digital circuit for the Boltzmann machine learning rules. Brause introduces the building blocks of a linear neuron that uses anti-Hebb rule and restricted weights. Raffo et al present a set of functional primitives that perform synaptic and neural functions which are implemented on an analog circuit.

\section{Digital Implementations of Neural Networks}

Digital implementations of neural networks provide some advantages, such as: noise free, programmability, higher precision, reliable storage devices. Delgado-Frias et al present a pipelined bit-serial digital organization that is able to implement a backpropagation learning algorithm. Fornaciari and Salice propose a tree-structure architecture based on a pseudo-neuron approach. Viredaz et al introduce a multi-model neural network computer based on the systolic computing paradigm. Asonovic et al present a fully programmable single chip microprocessor that is intended to be used within a Sun Sparcstation. Ae et al extend the self-organizing system (or Kohonen network) for semantic applications. Hui et al present a probabilistic RAM-based model that incorporates a reward-penalty learning algorithm. Hurdle et al propose an asynchronous design approach for neurocomputing; the CMAC neural model is used to demonstrate the approach capabilities.

\section{Neural Networks on Multiprocessor Systems and Applications}

König and Glesner present an architecture and implementation of a scalable associative memory system. Ryan et al describe how neural network can be mapped onto dataflow computing approach. A custom associative chip that has 64 fully interconnected binary 
neurons with on-chip leaming is presented by Gascuel et al The use of RISC processors as implementation of neural network simulation is a study by Rückert et al; they report results from eight different processors. Kolcz and Allinson have studied the implementation of CMAC, a perceptron-like computational structure, using conventional RAMs. Wang and Tang have developed GENET which is a competitive neural network model for AI's constraint satisfaction problems. Luk et al introduce a declarative language, called Ruby, that could be used in developing of neural network hardware. Palm et al present a study of the integration of connectionist models and symbolic knowledge processing. Styblinski and Minick present two Tank/Hopfield-like neural network methods for solving linear equations.

\section{VLSI Machines for Artificial Intelligence}

Eight papers address a number of current concerns in the hardware support of artificial intelligence processing. Lavington et al present a SIMD approach for pattern matching that is often used in production systems. Howe and Asonovic introduce a system that incorporates 148X36-bit content addressable parallel processors. Cannataro et al explain a message-passing parallel logic machine that can exploit AND/OR parallelism for logic programs. Rodohan and Glover outline an alternative search mechanism that can be implemented on the Associative String Processor. De Gloria et al present two performance analysis of a VLIW architecture where global compaction techniques are used. Civera et al discuss the application of a VLSI prolog system to real time navigation system; requirements, such as processes, memory bandwidth, and inferences per second, are presented. Demarchi et al present the design and a performance evaluation of a parallel architecture for OR-parallel logic programs. Yokota and Seo present a RISC Prolog processor which include compound instructions and dynamic switch mechanisms.

\section{ACKNOWLEDGMENTS}

This book is an edited selection of the papers presented at the International Workshop on VLSI for Neural Networks and Artificial Intelligence which was held at the University of Oxford in September 1992. Our thanks go to all the contributors and especially to the programme committee for all their hard work. Thanks are also due to the ACM-SIGARCH, the IEEE Computer Society, and the IEE for publicizing the event and to the University of Oxford and SUNY-Binghamton for their active support. We are particularly grateful to Laura Duffy, Maureen Doherty and Anna Morris for coping with the administrative problems. 


\section{CONTENTS}

\section{ANALOG CirCUITS FOR NEURAL NETWORKS}

Analog VLSI Neural Learning Circuits -A Tutorial

Howard C. Card

An Analog CMOS Implementation of a Kohonen Network with

Learning Capability

Oliver Landolt

Back-Propagation Learning Algorithms for Analog VLSI Implementation

Maurizio Valle, Daniele D. Caviglia and Giacomo M. Bisio

An Analog Implementation of the Boltzmann Machine with Programmable

Learning Algorithms

V. Lafargue, P. Garda and E. Belhaire

A VLSI Design of the Minimum Entropy Neuron

Rüdiger W. Brause

A Multi-Layer Analog VLSI Architecture for Texture Analysis Isomorphic to Cortical Cells in Mammalian Visual System

Luigi Raffo, Giacomo M. Bisio, Daniele D. Caviglia, Giacomo Indiveri and Silvio P. Sabatini

\section{DIGITAL IMPLEMENTATIONS OF NEURAL NETWORKS}

A VLSI Pipelined Neuroemulator

José G. Delgado-Frias, Stamatis Vassiliadis, Gerald G. Pechanek, Wei Lin,

Steven M. Barber and Hui Ding

A Low Latency Digital Neural Network Architecture

William Fornaciari and Fabio Salice

MANTRA: A Multi-Model Neural-Network Computer

Marc A Viredaz, Christian Lehmann, François Blayo and Paolo Ienne

Krste Asanovic', James Beck, Brian E. D. Kingsbury, Phil Kohn, Nelson Morgan and John Wawrzynek

Design of Neural Self-Organization Chips for Semantic Applications

Tadashi Ae, Reiji Aibara and Kazumasa Kioi

VLSI Implementation of a Digital Neural Network with Reward-Penalty Learning

Terence Hui, Paul Morgan, Hamid Bolouri and Kevin Gurney

Asynchronous VLSI Design for Neural System Implementation

John F. Hurdle, Erik L. Brunvand and Lüli Josephson

\section{NEURAL NETWORKS ON MULTIPROCESSOR SYSTEMS AND APPLICATIONS}

VLSI-Implementation of Associative Memory Systems for Neural

Information Processing

Andreas König and Manfred Glesner

A Dataflow Approach for Neural Networks

Thomas F. Ryan, José G. Delgado-Frias, Stamatis Vassiliadis,

Gerald G. Pechanek and Douglas M. Green

A Custom Associative Chip Used as a 3uilding Block for a Software Reconfigurable Multi-Network Simulator

Jean-Dominique Gascuel, Eric Delaunay, Lionel Montoliu, Bahram Moobed and Michel Weinfeld 
Parallel Implementation of Neural Associative Memories on RISC Processors

U. Rückert, S. Rüping and E. Naroska

Reconfigurable Logic Implementation of Memory-Based Neural Networks:

A Case Study of the CMAC Network

Aleksander R. Kolcz and Nigel M. Allinson

A Cascadable VLSI Design for GENET

Chang J. Wang and Edward P. K. Tsang

Parametrised Neural Network Design and Compilation into Hardware

Knowledge Processing in Neural Architecture

G. Palm, A Ultsch, K. Goser and U. Rückert

$M$. A. Styblinski and Jill R. Minick

\section{VLSI MACHINES FOR ARTIFICIAL INTELLIGENCE}

Hardware Support for Data Parallelism in Production Systems

$S$. H. Lavington, C. J. Wang, N. Kasabov and S. Lin

SPACE: Symbolic Processing in Associative Computing Elements

Denis B. Howe and Krste Asanovic'

PALM: A Logic Programming System on a Highly Parallel Architecture Mario Cannataro, Giandomenico Spezzano and Domenico Talia

A Distributed Parallel Associative Processor (DPAP) for the Execution of Logic Programs

Darren P. Rodohan and Raymond J. Glover

Performance Analysis of a Parallel VLSI Architecture for Prolog

Alessandro De Gloria, Paolo Faraboschi and Mauro Olivieri

A Prolog VLSI System for Real Time Applications

Pier Luigi Civera, Guido Masera and Massimo Ruo Roch

An Extended WAM Based Architecture for OR-Parallel Prolog Execution

Danilo Demarchi, Gianluca Piccinini and Maurizio Zamboni

Architecture and VLSI Implementation of a Pegasus-II Prolog Processor Takashi Yokota and Kazuo Seo

CONTRIBUTORS

INDEX 